\title{
A SIMPLIFIED 3-D FINITE ELEMENT METHOD APPLIED TO MODAL ANALYSES OF AN ELASTIC SURFACE LAYER
}

\author{
By Takumi TOSHINAWA* and Tatsuo OHMACHI**
}

\begin{abstract}
A simplified 3-D finite element method is newly proposed in order to simulate seismic wave propagation in a vast sedimentary basin. Eigenvalue analyses for $2-\mathrm{D}$ and $3-\mathrm{D}$ ground models with Poisson's ratio 0.25 or 0.40 are carried out to check validity of the method. The 2-D analyses demonstrate that Love wave modes can be accurately calculated in both cases. Rayleigh wave modes can also be calculated satisfactorily except for those with shorter wavelength and Poisson's ratio 0.40 . In $3-\mathrm{D}$ eigenvalue analyses by the present method, computational time is reduced to $1 / 170$ of the similar analyses by a conventional method.
\end{abstract}

Keywords:3-D, FEM, surface wave, eigenvalue analysis

\section{INTRODUCTION}

For the sake of mitigation of earthquake disaster in urban area on a sedimentary layer, prediction of ground motion induced by surface wave propagation is indispensable. This is because surface waves are easily generated and amplified in a soft sedimentary basin on which many metropolises are located. In addition, predominant periods of these waves are sometimes comparable to natural periods of large scale structures such as skyscrapers and long-span bridges.

To achieve the reliable prediction, numerical analysis using the finite element method ${ }^{1)}$ and the finite difference method $^{2)}$ have been recently used as a result of great progress in execution speed and storage capacity of electronic computers. Regarding analysis of vast ground, however, there still remains practical restriction because its analytical model will require a large number of degrees of freedom. To cope with this restriction, a simplified 2-D finite element method has been proposed for simulating Love wave propagation $^{3)}$. By extending this method, a simplified 3-D finite element has been formulated with a successful application to an eigenvalue analysis and an impulsive response analysis ${ }^{4)}$.

As for the southern part of the Kanto plain, Japan, the $\mathrm{P}$ and $\mathrm{S}$ wave velocities are respectively $1.8 \mathrm{~km}$ and $0.7 \mathrm{~km}$ for the surficial layer, and $5.6 \mathrm{~km}$ and $3.0 \mathrm{~km}$ for the seismic bedrock ${ }^{5}$. The corresponding Poisson's ratio ranges from 0.25 to 0.40 . On this basis, this paper deals with $2-\mathrm{D}$ off plane, in plane, and 3-D eigenvalue analyses using Poisson's ratio 0.25 and 0.40.

* Member of JSCE, M. Eng., Research Associate, Tokyo Institute of Technology (4259, Nagatsuta, Midori-ku, Yokohama)

** Member of JSCE, Dr. Eng. , Professor, Tokyo Institute of Technology (Ditto) 


\section{FORMULATION OF ELEMENT MATRICES}

\section{(1) Finite element modeling}

In this method, the ground is idealized as a single layer underlain by a half space. The finite element modeling of the ground is done by using triangular prismatic elements as shown in Fig. 1. Fig. 2 shows one of the elements whose six nodal points are numbered counterclockwise. The prismatic element in a global Cartesian co-ordinate can be simply expressed by a local co-ordinate shown in Fig. 3. In terms of the local co-ordinates and interpolation functions, displacements in the elements are expressed as follows;

$$
u=\sum_{i=1}^{6} N_{i} u_{i}, \quad v=\sum_{i=1}^{6} N_{i+6} v_{i}, \quad w=\sum_{i=1}^{6} N_{i+12} w_{i} \cdots
$$

where

$$
\begin{aligned}
& N_{1}=\xi_{1} f(\zeta), \quad N_{2}=\xi_{2} f(\zeta), \quad N_{3}=\xi_{3} f(\zeta) \\
& N_{4}=\xi_{1}\{1-f(\zeta)\}, \quad N_{5}=\xi_{2}\{1-f(\zeta)\}, \quad N_{6}=\xi_{3}\{1-f(\zeta)\} \\
& N_{7}=\xi_{1} g(\zeta), \quad N_{8}=\xi_{2} g(\zeta), \quad N_{9}=\xi_{3} g(\zeta) \\
& N_{10}=\xi_{1}\{1-g(\zeta)\}, \quad N_{11}=\xi_{2}\{1-g(\zeta)\}, \quad N_{12}=\xi_{3}\{1-g(\zeta)\} \\
& N_{13}=\xi_{1} h(\zeta), \quad N_{14}=\xi_{2} h(\zeta), \quad N_{15}=\xi_{3} h(\zeta) \\
& N_{16}=\xi_{1}\{1-h(\zeta)\}, \quad N_{17}=\xi_{2}\{1-h(\zeta)\}, \quad N_{18}=\xi_{3}\{1-h(\zeta)\}
\end{aligned}
$$

$u, v$, and $w$ represent displacements in the $x, y$, and $z$ co-ordinates, and $f(\zeta), g(\zeta)$, and $h(\zeta)$ are interpolation functions for $u, v$, and $w$ along the $z$ axis. These interpolation functions are normalized to satisfy the following conditions;

$f(1)=g(1)=h(1)=1, \quad f(-1)=g(-1)=h(-1)=0$

Then shape functions for strain and displacement can respectively be expressed in the following matrix forms

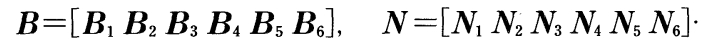

in which

$$
\boldsymbol{B}_{i}=\left[\begin{array}{ccc}
\partial N_{i} / \partial x & 0 & 0 \\
0 & \partial N_{i+6} / \partial y & 0 \\
0 & 0 & \partial N_{i+12} / \partial z \\
\partial N_{i} / \partial y & \partial N_{i+6} / \partial x & 0 \\
0 & \partial N_{i+6} / \partial z & \partial N_{i+12} / \partial y \\
\partial N_{i} / \partial z & 0 & \partial N_{i+12} / \partial x
\end{array}\right] \quad(i=1,6), \quad N_{i}=\left[\begin{array}{ccc}
N_{i} & 0 & 0 \\
0 & N_{i+6} & 0 \\
0 & 0 & N_{i+12}
\end{array}\right] \quad(i=1,6) \ldots \ldots \ldots
$$

Finally, we have the stiffness and mass matrices;

$$
\boldsymbol{k}=\int_{v} \boldsymbol{B}^{T} \boldsymbol{D} \boldsymbol{B} \mathrm{d}(\mathrm{vol})
$$
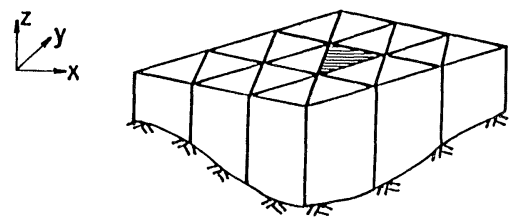

Fig. 1 Finite Element Modeling of 3-D Ground.
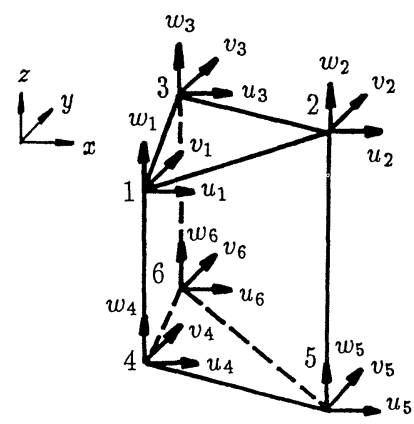

Fig. 2 A Triangular Prismatic Element.

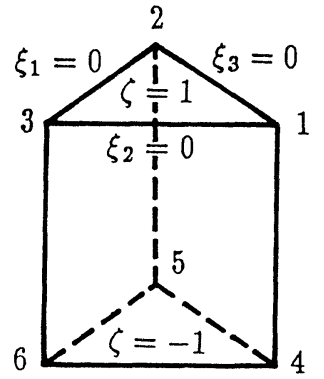

Fig. 3 A Prismatic Element in a Local Co-ordinate. 


$$
\boldsymbol{m}=\rho \int_{v} \boldsymbol{N}^{T} \boldsymbol{N} \mathrm{d}(\mathrm{vol})
$$

where $\boldsymbol{D}$ indicates the three dimensional elasticity matrix. Although a consistent mass matrix is obtained from Eq. (7), we adopted a lumped mass matrix for the sake of computational convenience. Diagonal terms of this matrix are set proportional to corresponding diagonal terms of the consistent matrix. The proportional constant was determined from conditions for preserving total mass of the element. By trial and error, we found this matrix being superior to the lumped matrix obtained by adding off-diagonal terms to diagonal ones of the consistent matrix. The integrals in Eqs. (6) and ( 7 ) are readily evaluated by the Gaussian quadrature, the integral points of which are three points in the $\xi_{1}-\xi_{2}-\xi_{3}$ plane and four points along the $\zeta$ axis. The number of integral points along the $\zeta$ axis was determined by checking accuracy of integrations of sinusoidal functions such as $\sin \pi \zeta / 2$ and $\sin 3 \pi \zeta / 2$. By assembling the element matrices, total mass and stiffness matrices can be obtained.

\section{TWO DIMENSIONAL EIGENVALUE ANALYSES}

\section{(1) 2-D ground model}

To check the validity of this method, the computational program was tested through 2-D eigenvalue analyses. Fig. 4 shows ground model employed for the analyses. This model is $1 \mathrm{~km}$ deep, $8 \mathrm{~km}$ long, and $1 / 6 \mathrm{~km}$ wide, and is devided into 48 pseudo elements along the $x$-axis (a pseudo element is made of two prismatic elements). Shear wave velocity, mass density, and Poisson's ratio are $0.7 \mathrm{~km} / \mathrm{s}, 1.9 \mathrm{t} / \mathrm{m}^{3}$, and 0.25 or 0.40 , respectively. Two types of anlyses were conducted; that is, the Love wave case and Rayleigh wave case. In the Love wave case, all motions are perpendicular to the $x-z$ plane, and in the Rayleigh wave case, all displacements occur in the $x-z$ plane. Boundary conditions for both cases are tabulated in Table 1. Regarding Rayleigh waves, horizontal motion has $\pi / 2$ difference in phase from vertical one. This is why only horizontal displacement is allowed at the edge in the Rayleigh wave case. Therefore, the number of degrees of freedom in the Love wave case is 94 , and that in the Rayleigh wave case is 192. As more than eight elements are necessary for any wavelength to secure accuracy of the solution $^{7)}$, modal vectors expressing wavelength shorter than $4 / 3 \mathrm{~km}$ are considered less accurate. Despite Poisson's ratio, the mode shapes of Rayleigh waves in an elastic medium on a rigid half space are almost similar, when the dimensionless wavelength which is defined as a ratio of wavelength to thickness of the layer is longer than $6^{8}$. Besides, the mode shapes for horizontal motion of Rayleigh waves are similar to those of Love waves. On this basis, the Rayleigh wave mode shapes at the dimensionless wavelength of 10 are employed for the interpolation functions. As shown in Fig. 5 in which the lateral lines denote the Gaussian integral points, these functions satisfy the boundary conditions in Eq. (3). Ground motions made up of many modes can be obtained by superposing each modal motion separately calculated with a set of three shape functions.

(2) Love wave case

Fig. 6 shows analytical examples whose dimensionless wavelengths are 16/3. From eigensolutions, phase velocity are obtained by ${ }^{9)}$

$c=\lambda / f$

Table 1 Boundary Conditions of the 2-Dimensional Model.

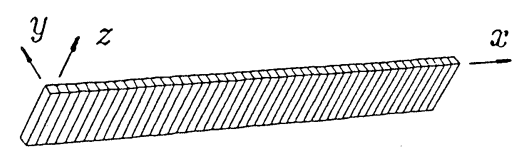

Fig. 4 2-D Ground Model.

\begin{tabular}{|c|c|c|c|}
\hline & at $x=0, L$ & at $z=-H$ & other points \\
\hline & $x:$ fixed & $x:$ fixed & $x:$ fixed \\
\hline \multirow[t]{2}{*}{ Love wave case } & $y:$ fixed & $y:$ fixed & $y:$ fixed \\
\hline & $z:$ fixed & $z:$ fixed & $z:$ fixed \\
\hline \multirow{3}{*}{ Rayleigh wave case } & $x:$ free & $x:$ fixed & $x$ :free \\
\hline & $y:$ fixed & $y:$ fixed & $y:$ fixed \\
\hline & $z$ :fixed & $z: f i x e d$ & $z$ free \\
\hline
\end{tabular}




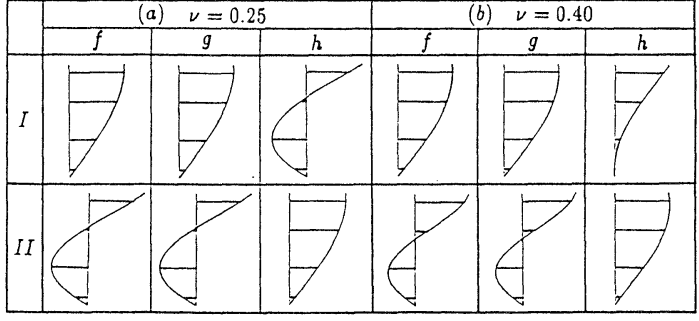

Fig. 5 Interpolation Functions Employed for Analyses.

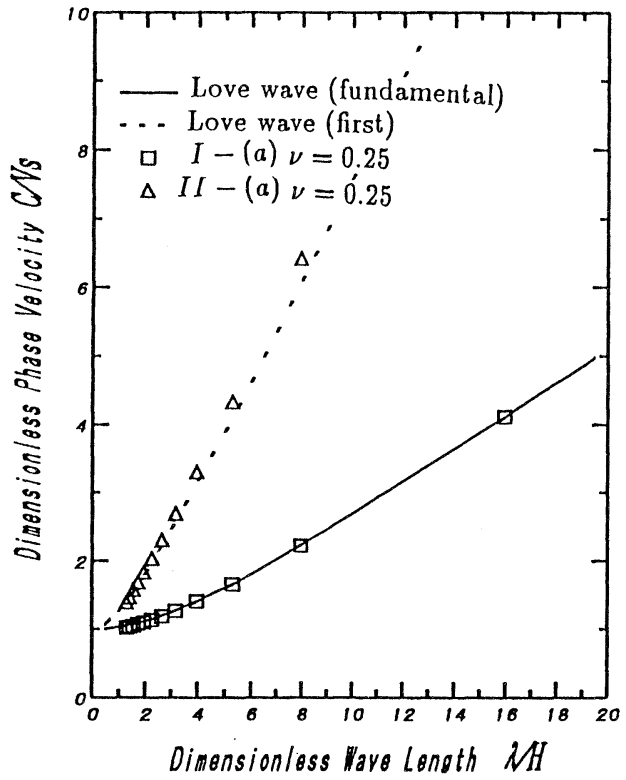

(a) $\nu=0.25$

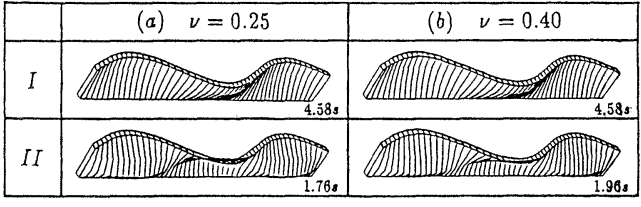

Fig. 6 Eigenvectors in Love Wave Case $(\lambda / H=5.33)$.

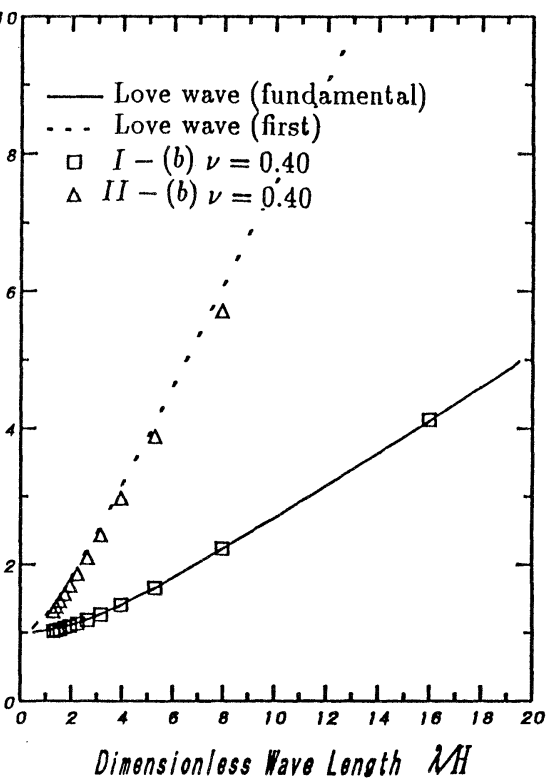

(b) $\quad \nu=0.40$

Fig. 7 Characteristics of Phase Velocity Dispersion in Love Wave Case.

in which $c, \lambda$, and $f$ are phase velocity, wavelength, and natural frequency, respectively. Dimensionless quantities $\lambda / H$ and $C / V s$ are obtained by referring to the shear wave velocity $V s$ and thickness of the layer $H$. Fig. 7 shows the analytical results of the Love wave case expressed

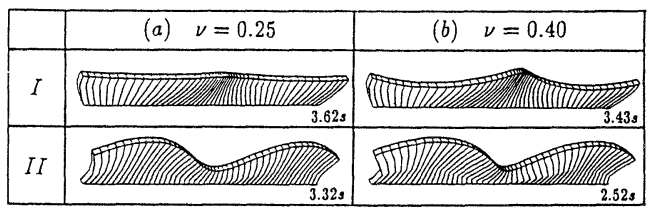

Fig. 8 Eigenvectors in Rayleigh wave case $(\lambda / H=5.33)$. in terms of dimensionless phase velocity against dimensionless wavelength. Hollow squares and triangles in these figures indicate those resulting from functions I and II in Fig. 5, respectively. For the sake of comparison, dispersion curves for fundamental and first higer mode Love waves are also drawn in these figures by solid and broken lines. A set of functions $I$ is found to give good agreement with theoretical values. This is because the functions employed for $f(\zeta)$ and $g(\zeta)$ are almost coincident with eigenfunctions for the fundamental mode shapes of Love waves. A set of functions II causes a little discrepancy because of their slight difference from eigenfunctions for the first higer mode shapes of Love waves.

\section{(3) Rayleigh wave case}

Fig. 8 shows some examples in the Rayleigh wave case. The phase velocity dispersion characteristics are shown in Fig. 9. Hollow squares and triangles in the figure were resulted from two sets of functions I and II shown in Fig. 5. Solid and broken lines in Fig. 9 are $M_{11}$ and $M_{21}$ Rayleigh wave dispersion curves, respectively. Fig. 10 shows the ratios $U / W$ of the horizontal amplitude to the vertical one at the free 


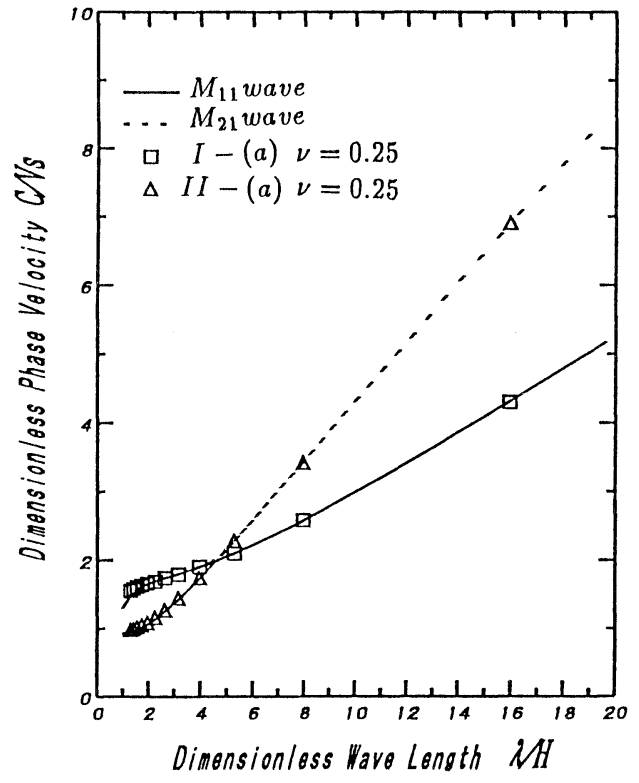

(a) $\nu=0.25$

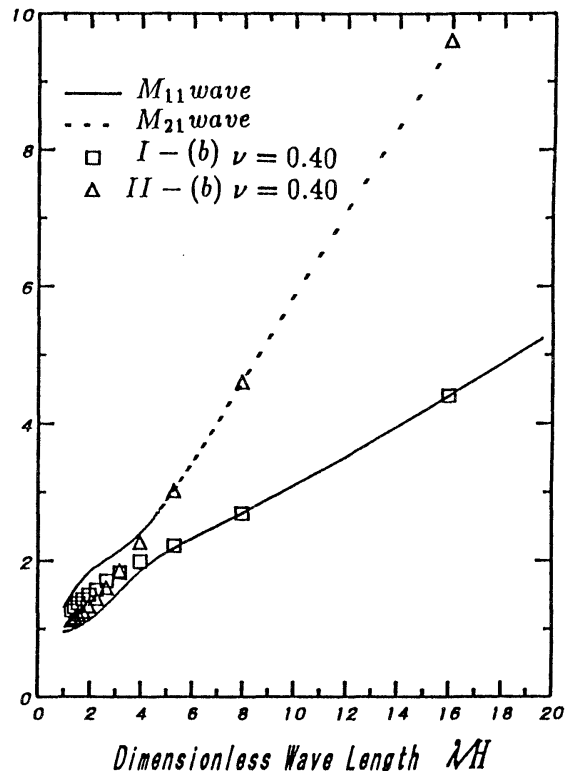

(b) $\nu=0.40$

Fig. 9 Characteristics of Phase Velocity Dispersion in Rayleigh Wave Case.
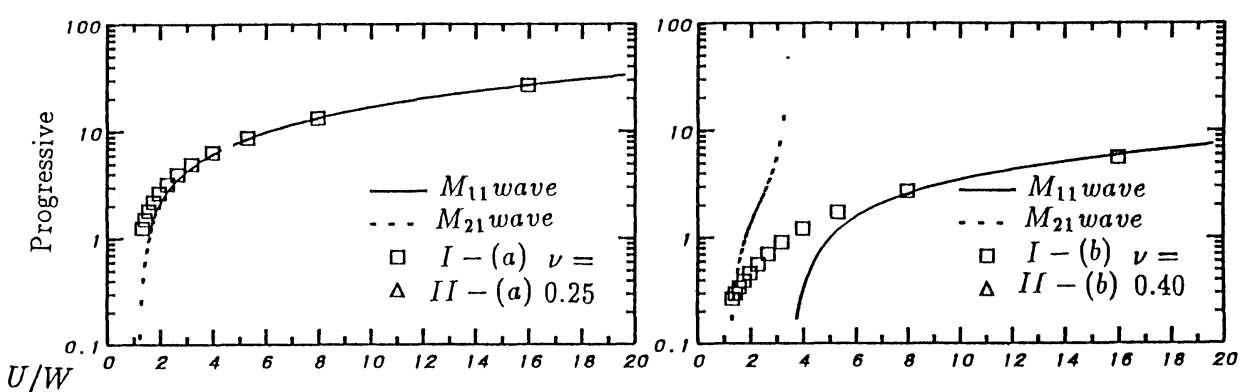

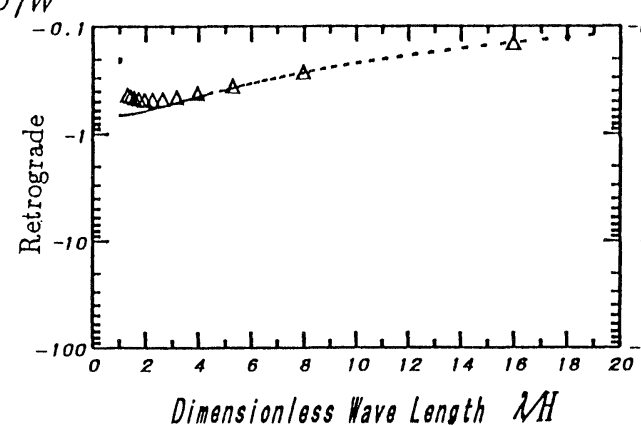

(a) $\nu=0.25$

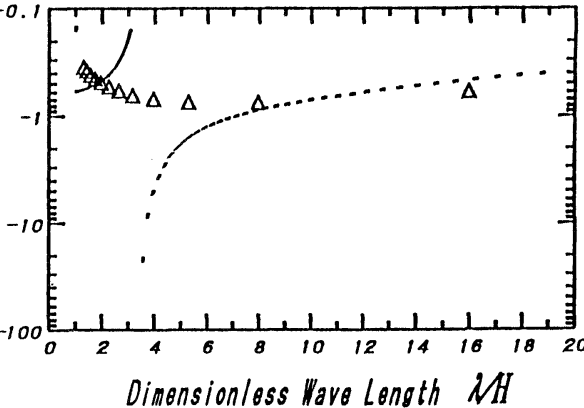

(b) $\nu=0.40$

Fig. 10 Amplitude Ratio in Rayleigh Wave Case.

surface, and solid and broken lines show theoretical curves. Rotational direction of the particle motion can be found from these values; that is, progressive for the positive values, and retrograde for the negative ones $^{10)}$. The direction of the motion can also be seen from the calculated modal vectors because the vectors are equivalent to stationary waves consisting of a pair of waves traveling in the positive $x$ and negative $x$ directions. It is evident that the calculated characteristics such as phase velocity dispersion and displacement ratio are different from the theoretical ones only when dimensionless wavelength is shorter 
than 5 in case of Poisson's ratio 0.40 . The results show that the present procedure is better adopted to the analyses with Poisson's ratio 0.25.

\section{THREE DIMENSIONAL EIGENVAL- UE ANALYSES}

\section{(1) 3-D ground model}

The above-mentioned 2-D eigenvalue analysis has demonstrated validity of the present method in

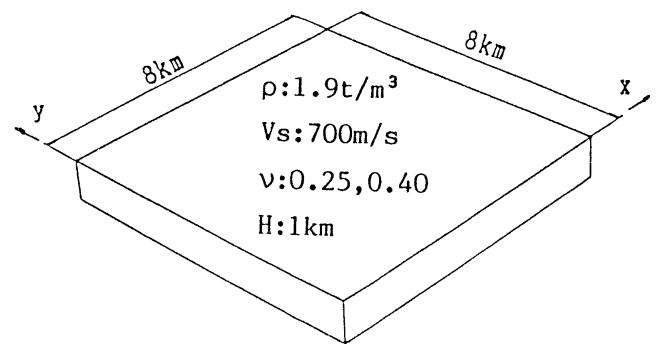

Fig. 11 3-D Ground Model. the $\mathrm{SH}$ and the P-SV fields, separately. In order to investigate the applicability to 3-D field, 3-D eigenvalue analysis is performed using a simple 3-D model which is obtained by expanding the width of the 2-D model in the $y$ direction. No displacement is allowed at the side walls and at the bottom, whereas diplacement in three directions are allowed inside the walls. Material properties of the model are the same with those of the 2-D model. The interpolation functions used here are those of $\mathrm{I}^{-}(\mathrm{a})$ and $\mathrm{I}^{-}(\mathrm{b})$ in Fig. 5. To compare the accuracy and advantage of this method, a conventional 3-D finite element method which uses the tetrahedral elements ${ }^{11)}$ is applied to the same eigenvalue analysis, because in our thinking, the tetrahedral element has been most popular in 3-D finite element analyses.
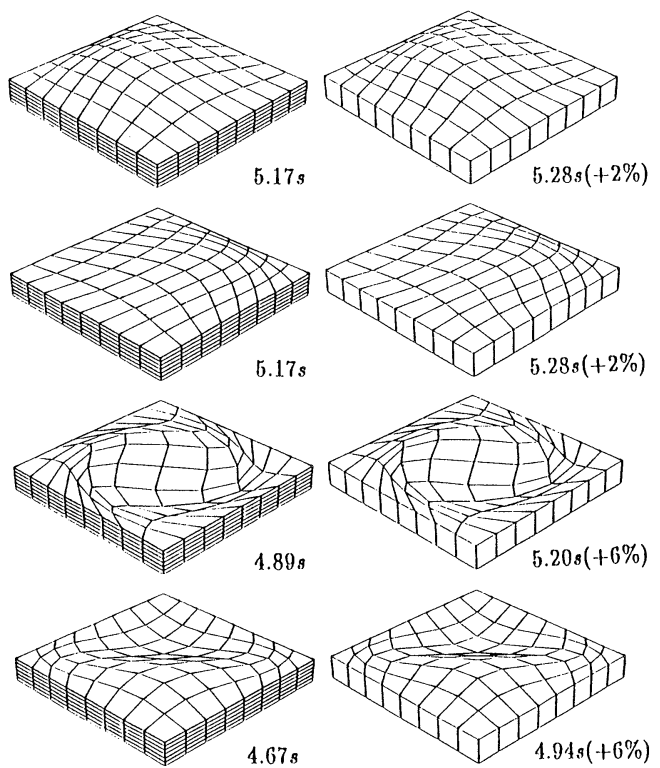

by

conventional method

(a) $\nu=0.25$ by

present method

Fig. 12 Comparison of 3-D Eigenvectors.

by
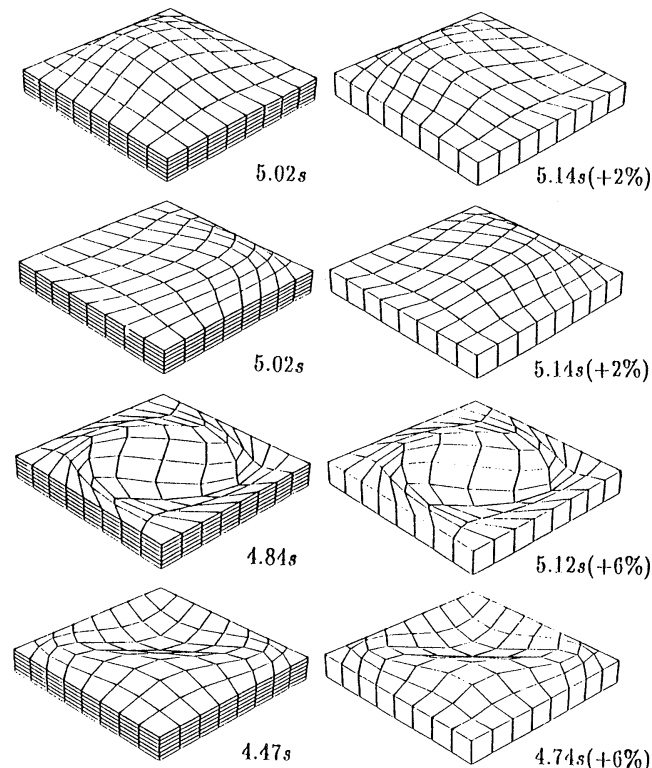

conventional method

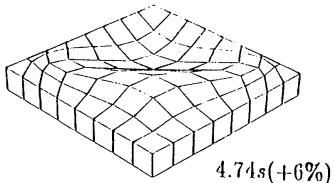

by

present method

(b) $\nu=0.40$

Table 2 Comparison of Analytical Qualities.

\begin{tabular}{lccc}
\hline & A: Conventional Method & B: Present Method & $B / A$ \\
\hline Number of nodes & 567 & 162 & $1 / 3.5$ \\
\hline N.D.O.F. & 882 & 147 & $1 / 6$ \\
\hline Elapsed cpu time & $1000 \mathrm{~s}$ & $6 \mathrm{~s}$ & $1 / 167$ \\
\hline
\end{tabular}




\section{(2) Comparison with results from a conventional method}

a) Eigenvectors and eigenvalues

The lowest four modes obtained by the analysis are shown in Fig. 12. In the first and second modes, horizontal motion toward the outside directions is predominant with a little vertical motion. The next higer mode is the torsional mode, and the fourth mode is the dilatational mode in the diagonal direction. In the analysis with Poisson's ratio 0.40 , the directions of motion in the first and second modes calculated by the present method are of a little difference from those of the conventional method, whereas the shapes in the third and fourth modes are much similar to each other. In the analysis with Poisson's ratio 0.25, the eigenmodes look coincident with each other. The present procedure tends to give natural periods longer than those by the conventional FEM. The difference in period is $2 \%$ for the first and second mode, and $6 \%$ for the third and fourth mode.

b) Computational time

Number of nodes, N. D. O.F. (number of degrees of freedom) and the elapsed cpu time to calculate the lowest 30 modes are tabulated in Table 2. It is evident that the computational task can be much reduced by this method in comparison with the conventional one.

\section{CONCLUSIONS}

The finite element matrices have been formulated by using the modal shape functions of Rayleigh waves for the interpolation functions. The matrices enable us to reduce the number of degrees of freedom to a great extent. As far as a series of eigenvalue analyses demonstrated here is concerned, the following conclusions can be drawn with respect to the present method.

(1) Dispersion characteristics of fundamental Love wave can be accurately calculated.

(2) When Poisson's ratio is 0.25 , Rayleigh wave characteristics such as phase velocity dispersion and amplitude ratio can be calculated to a satisfactory level of accuracy in the full range of wavelength.

(3) When Poisson's ratio is 0.40 , the calculated Rayleigh wave characteristies are reliable except in a shorter wavelength range.

(4) Computational time can be reduced to $1 / 170$ with sufficient accuracy in 3-D eigenvalue analysis. In this study, a rigid half space was assumed for the second layer. We are planning to analyze seismic wave propagation in more generalized ground models by extending this method.

\section{ACKNOWLEDGEMENTS}

The numerical computation for this study was performed on the HITAC M-660 K of Computer Center, Tokyo Institute of Technology.

\section{REFERENCES}

1) Ohtsuki, A. and Harumi, K. : Effect of topography and subsurface inhomogeneities on seismic SV waves, Int. J. Earthquake Engrg. Struct. Dyn. 11, pp. 441-462, 1983.

2) Vidale, J.E. and Helmberger, D. V. : Elastic finite-difference modeling of the 1971 San Fernando, California earthquake, Bulletin of the Seismological Society of America, Vol.78, pp. 122-141, 1988.

3) Ohmachi, T. and Toshinawa, T. : A simplified numerical analysis procedure for surface waves propagating in sedimentary layers, Proceedings of 9 th Conference on Earthquake Engineering, Vol. II, pp. 635-640, 1988.

4) Toshinawa, T. and Ohmachi, T. : Numerical analysis technique for surface wave propagation problem in a vast $3-\mathrm{D}$ ground, Proceedings of the 20 th JSCE Earthquake Engineering Symposium, pp. 201-204, 1989 (in Japanese).

5) Yamanaka, H. et al. : On the seismic prospecting in the southwestern part of the Tokyo metropolitan area ( 3 ), Bulletin of Seismological Society of Japan, Vol.41, pp. 527-539, 1988 (in Japanese).

6) Morichi, S. et al. : Vibration modes characterized by Rayleigh waves propagating in an elastic layer on a rigid base, Proc. of JSCE, Structural Eng. /Earthquake Eng., Vol. 4, No. 1, pp. 191-199, April, 1987.

7) Kuhlemeyer, R. L. and Lysmer, J. : Finite element accuracy for wave propagation problems, Journal of the Soil Mechanics and Foundations Division, Proceedings of ASCE, Vol.99, No. SM 5, pp. 421-427, May, 1973. 
8) Ohta, Y. : Rayleigh type dispersive waves in stratified double layer (II), Bulletin of Seismological Society of Japan, Vol. 17, pp. 89-100, 1964 (in Japanese).

9) Morichi, S., Ohmachi, T. and Toshinawa, T. : Vibration modes characterized by Love waves in an elastic layer overlying a rigid basement, Proc. of JSCE, Structural Eng./Earthquake Eng., Vol. 2, No. 1, pp. 227-235, April, 1985.

10) Ohta, Y. : Particle motion of Rayleigh type dispersive waves, Bulletin of Seismological Society of Japan, Vol. 17, pp. 179-186, 1964 (in Japanese).

11) Zienkiewicz, O. C. : The finite element method, Third Edition, McGraw-Hill Book Company, pp. 178-199, 1977.

(Received October 11 1989) 